\title{
Supersymmetric Wilson loops with general contours in ABJM theory
}

\author{
Nakwoo Kim田 \\ Department of Physics and Research Institute of Basic Science, \\ Kyung Hee University, 1 Hoegi-dong, \\ Dongdaemun-gu, Seoul 130-701, Korea
}

\begin{abstract}
We consider general supersymmetric Wilson loops in ABJM model, i.e. Chern-Simons-matter theory in $2+1$ dimensions with $\mathcal{N}=6$ supersymmetry. They are so-called Zarembo-type: the Wilson loops of our interest have generic contours in spacetime, but the scalar field coupling is arranged accordingly so that there are unbroken supersymmetries. Based on the supermatrix formulation of Wilson loops by Drukker and Trancanelli, we construct explicitly 1/6-BPS Wilson loops and check that their expectation value is protected using perturbation up to two loops. We also study the dual string configuration in $A d S_{4} \times \mathbb{C P}^{3}$ background and check the supersymmetry.
\end{abstract}

PACS numbers: 11.15.Yc, 11.25.Tq,11.25.Db

Keywords: Wilson loops, Chern-Simons theory, AdS/CFT

*Electronic address: nkim@khu.ac.kr 


\section{INTRODUCTION}

Wilson loops are essential objects in the study of AdS/CFT correspondence [1]. On the gauge theory side they provide gauge singlet operators whose vacuum expectation value characterizes the confining/deconfining phase. On the string theory side they are simply fundamental strings, and their classical solution is given as minimal surface [2] 3]. The importance of Wilson loops in AdS/CFT partly stems from the fact that they are amenable to both perturbative gauge theory computation [4] and a string theory computation in the region of large coupling constant [2] 3]. It is also possible to calculate the expectation value of supersymmetric Wilson loops exactly using the technique of localization [5].

In this paper we are interested in supersymmetric Wilson loops in $\mathcal{N}=6$ Chern-Simonsmatter theory in $2+1$-dimensions. This theory was proposed as the gauge theory on M2branes put on orbifold singularity $\mathbb{R}^{8} / \mathbb{Z}_{k}$ by Aharony, Bergman, Jafferis, and Maldacena (ABJM) [6]. See also the Aharony-Bergman-Jafferis (ABJ) model [7] of fractional M2branes which have different gauge group ranks, i.e. $S U(N) \times S U(M)$. In the large coupling region the relevant gravity dual background is $A d S_{4} \times \mathbb{C P}^{3}$ in IIA string theory, and the Wilson loop variables are expected to be dual to fundamental strings. Initially there was a puzzle over the matching of gravity side and gauge theory side description for Wilson loops: On gravity side a simple straight fundamental string is $1 / 2$-BPS, while constructing 1/2-BPS Wilson line in ABJM turned out to be nontrivial [8] [9] [10].

This enigma was resolved by Drukker and Trancanelli [11] when they constructed explicitly 1/2-BPS Wilson loops in ABJM model using supermatrix. In contrast to the supersymmetric Wilson loops in $\mathcal{N}=4$ super Yang-Mills theory, the Wilson loops in [1] have couplings to fermion fields in bi-fundamental representation of the gauge group $U(N) \times U(N)$. The authors of [11] considered Wilson lines which are straight lines or circular loops. They are all 1/2-BPS, and are related through conformal transformation.

Our aim here is to provide a nontrivial test of AdS/CFT for ABJM model, by considering supersymmetric Wilson loops with generic contours. Such objects in $\mathcal{N}=4$ super YangMills theory were first constructed by Zarembo [12]. One important feature of Zarembo-type Wilson loop is that the scalar field coupling is coordinated with the spacetime contour so that there is unbroken supersymmetry. If the contour is straight line it is $1 / 2$-BPS, while if the loop is embedded in $\mathbb{R}^{2}\left(\mathbb{R}^{3}\right)$ it is $1 / 4$-BPS $(1 / 8$-BPS). 
The study of less-than-half BPS Wilson loops in ABJM theory was initiated in [13]. Cusp-like, or piecewise linear Wilson lines are studied and it was explicitly checked that for planar ones the supersymmetry is broken to $1 / 6$. Then the extension to Zarembo-type Wilson loops of arbitrary shape was presented in [14].

In this paper we aim to elaborate upon the result in [14]. In particular, we construct a Zarembo-type circular Wilson loop explicitly and check if its expectation value vanishes perturbatively, up to two loops. We also demonstrate the supersymmetry of the dual string configuration, using the extremal surface satisfying the pseudo-holomorphicity condition in [15].

We note that recent papers which study supersymmetric Wilson lines in ABJM model include [16 26]. For conventions and the Feynmann rules for perturbative computations we use in this paper, the readers are referred to [13].

This paper is organized as follows. In Sec II A we introduce the supermatrix Wilson loop formalism [11] and discuss the role of boundary condition for supersymmetry condition. In Sec.IIB we present the configuration of 1/6-BPS Wilson loops explicitly. Then in Sec.III we describe the perturbative computation of our Wilson loops and check it vanishes up to two-loops. In Sec IV we provide the dual picture and show the pseudo-holomorphic string solution is $1 / 6$-BPS using $\kappa$-symmetry projection. We then conclude in Sec.V.

\section{SUPERSYMMETRIC WILSON LOOPS IN ABJM THEORY}

\section{A. The supermatrix formalism and the boundary condition of Wilson loops}

Let us first recall the supermatrix Wilson loop of Drukker and Trancanelli [11]. The Wilson loop operator is given as a path-ordered exponential integral defined by a curve $x^{\mu}(\tau)$ in spacetime,

$$
W=\operatorname{Tr} \mathcal{P} \exp \left(\int L d \tau\right) .
$$

Here the exponentiated object $L$ is a supermatrix,

$$
L=\left(\begin{array}{cc}
i A_{\mu} \dot{x}^{\mu}+\frac{2 \pi}{k}|\dot{x}| M_{J}^{I} C_{I} \bar{C}^{J} & \sqrt{\frac{2 \pi}{k}}|\dot{x}| \eta_{I}^{\alpha} \bar{\psi}_{\alpha}^{I} \\
\sqrt{\frac{2 \pi}{k}}|\dot{x}| \psi_{I}^{\alpha} \bar{\eta}_{\alpha}^{I} & i \hat{A}_{\mu} \dot{x}^{\mu}+\frac{2 \pi}{k}|\dot{x}| \hat{M}_{I}^{J} \bar{C}^{J} C_{I}
\end{array}\right)
$$

We note that in this article the Wilson lines are defined along a space-like curve. When necessary, we will consider Euclidean $\mathbb{R}^{3}$ as the spacetime. The choice above is made to be 
consistent with this convention. As well-known, the ABJM model has gauge group $U(N) \times$ $U(N)$. More generally the Aharony-Bergman-Jafferis (ABJ) model [7], a Chern-Simons model which incorporates fractional M2-braneABJ model may have different ranks for the gauge group, i.e. $U(N) \times U(M)$. The above supermatrix $L$ is in general $(N+M) \times(N+M)$, and the top-left block is $N \times N$, etc. $k$ is the Chern-Simons coefficient.

In the above $A_{\mu}, \hat{A}_{\mu}$ represent the two gauge fields. $C_{I}, I=1,2,3,4$ are the bifundamental scalar fields and $N \times M . \bar{C}^{I}$ are their conjugate fields. The same applies to fermionic fields $\psi_{I}, \bar{\psi}^{I}$. On the other hand, $x, M, \hat{M}, \eta, \bar{\eta}$ are $\tau$-dependent parameters, defining the Wilson line. In particular, they encode the shape of the Wilson loop in spacetime $\left(x^{\mu}\right)$ or in the internal space $\mathbb{C P}^{3}(M$ and $\hat{M})$. Also note that the spinorial parameters $\eta, \bar{\eta}$ are commuting variables. Thus in the end the diagonal blocks are commuting, while the off-diagonal blocks are anti-commuting quantities.

Before we embark on the 1/6-BPS Wilson lines, let us make a comment on the relation between the boundary condition of the fields along the Wilson line and the choice of taking either ordinary trace or supertrace. For convenience let us record a few terms in the pathordered exponent.

$$
W=\operatorname{Tr} \mathbb{I}+\int_{-\infty}^{\infty} d \tau \operatorname{Tr} L+\int_{-\infty}^{\infty} d \tau_{1} \int_{-\infty}^{\tau_{1}} d \tau_{2} \operatorname{Tr} L\left(\tau_{1}\right) L\left(\tau_{2}\right)+\cdots
$$

When one considers the supersymmetric variation of $W$ for straight line with constant $M, \hat{M}, \eta, \bar{\eta}$ chosen appropriately, schematically the result is (see eq.(2.12) of [11] with $\eta \bar{\eta}=$ $2 i)$

$$
\delta W \propto \operatorname{Tr}\left[\int_{-\infty}^{\infty} d \tau\left(\begin{array}{ll}
C \psi & \\
& \psi C
\end{array}\right)-\int_{-\infty}^{\infty} d \tau_{1} \int_{\tau_{1}}^{\infty} d \tau_{2}\left(\begin{array}{ll}
\partial_{\tau_{1}} C\left(\tau_{1}\right) \psi\left(\tau_{2}\right) & \\
& -\psi\left(\tau_{1}\right) \partial_{\tau_{2}} C\left(\tau_{2}\right)
\end{array}\right)\right]
$$

Integrating the second term once, it is obvious that the bulk contribution cancels the first term for both top-left and bottom-right blocks. The boundary term of this integration was simply ignored in [11], but we would like to demand that they should cancel out eventually as well. It is easy to check that

$$
\delta W \propto \operatorname{Tr} \int_{-\infty}^{\infty} d \tau\left(\begin{array}{ll}
C(-\infty) \psi(\tau) & \\
& \psi(\tau) C(\infty)
\end{array}\right)
$$

If we impose the periodic boundary condition $C(-\infty)=C(\infty)$, which is obviously a natural choice for bosonic fields $C^{I}$, the variation is zero when we take the supertrace. In this 
paper we are interested in generic contour generalization of Drukker-Trancanelli 1/2-BPS Wilson lines with trivial expectation values. We may discretize such loops as a composite of infinitesimal line elements, and clearly taking supertrace one can preserve some supersymmetry with this convention. We discuss the remaining supersymmetry in more detail in the next sections.

\section{B. 1/6-BPS Wilson loop with generic contours}

\section{Argument using cusp Wilson lines}

Using the supermatrix representation above, Drukker and Trancanelli [11] showed that for a specific choice of $x^{\mu}, M_{J}^{I}$ the Wilson loop operator can be $1 / 2$-BPS. For the class of BPS operators with unbroken Poincaré supersymmetry, the Wilson line is defined along a straight line, e.g. $x^{\mu}=(0,0, \tau)$. The choice of $M, \hat{M}$ is made via a point in $\mathbb{C P}^{3}$. In other words,

$$
M_{J}^{I}=\hat{M}_{J}^{I}=-2 \frac{\bar{z}^{I} z_{J}}{|z|^{2}}+\delta_{J}^{I} .
$$

Then one can show that if we choose $z_{I}$ to be constant, e.g. $z=(1,0,0,0)$ and $M=$ $\hat{M}=\operatorname{diag}(-1,1,1,1)$ the Wilson loop preserves $1 / 2$ supersymmetry. The value of spinorial parameters $\eta, \bar{\eta}$ is also crucial, and as a $d=3$ spinor they are eigenspinors of $\dot{x}^{\mu} \gamma_{\mu}$ with normalization $\eta \bar{\eta}=2 i$. Note that, just as $M$ and $\hat{M}$ are in principle independent of each other, $\eta$ and $\bar{\eta}$ are independent and do not need to be conjugate to each other.

The ABJM model has $\mathcal{N}=6$ supersymmetry, and the transformation parameters can be written as $\theta_{\alpha}^{I J}$. Here $I, J$ are $S U(4)$ indices and antisymmetrized, and $\alpha=1,2$ are Dirac indices in $d=3$. For the particular 1/2-BPS Wilson loop operators above, the remaining supersymmetries are shown to be generated by the following set of parameters [11].

$$
\bar{\theta}_{+}^{12}, \bar{\theta}_{+}^{13}, \bar{\theta}_{+}^{14}, \bar{\theta}_{-}^{23}, \bar{\theta}_{-}^{34}, \bar{\theta}_{-}^{42}
$$

The rule for preserved supersymmetry is very simple: for $\bar{\theta}^{1 i} \dot{x}^{\mu} \gamma_{\mu}=\bar{\theta}^{1 i}, \quad \bar{\theta}^{j k} \dot{x}^{\mu} \gamma_{\mu}=$ $-\bar{\theta}^{j k}, i, j, k=2,3,4$ when $z^{I}=\delta_{1}^{I}$.

Now let us rationalize the existence of less supersymmetric Wilson loop operators, using a simple configuration. Consider another Wilson loop which is directed opposite to the one above in spacetime $\left(x^{\mu}=(0,0,-\tau)\right)$ and on a different point in $\mathbb{C P}^{3}, z=(0,1,0,0)$. Then 
obviously the supersymmetry should be now generated by

$$
\bar{\theta}_{-}^{21}, \bar{\theta}_{-}^{23}, \bar{\theta}_{-}^{24}, \bar{\theta}_{+}^{13}, \bar{\theta}_{+}^{34}, \bar{\theta}_{+}^{41},
$$

If we look at the intersection of these two sets, we realize it has four elements

$$
\bar{\theta}_{+}^{13}, \bar{\theta}_{+}^{14}, \bar{\theta}_{-}^{23}, \bar{\theta}_{-}^{24}
$$

In other words, the composite of these two Wilson lines preserve 4 out of 12 original Poincaré supersymmetry, i.e. $1 / 3$-BPS.

Of course one can glue them together as two half-lines, and obtain a Wilson loop with a cusp of deflection angle $\pi$. Although at first sight it looks like we have $\pi / 2$ rotation with $\zeta^{I}$, we should recall that they are in spinor representation of $S O(6) . z=(1,0,0,0)$ into $z=(0,1,0,0)$ corresponds to $\pi$ rotation in the $S U(2)$ subsector made of $z_{1}, z_{2}$, as multiplication of $\exp \left(-i \pi \sigma_{2} / 2\right)$.

In fact the Wilson lines which are piecewise linear with cusps are studied recently in [13. In those works Wilson lines on a plane are considered and it is checked that for a generic deflection angle the configuration is 1/6-BPS. For the supersymmetry of Wilson loops with generic contour $x(\tau)$, we can consider discretizing them into a collection of infinitesimal linear intervals. When the deflection angle, or the curvature in spacetime and in internal $\mathbb{C P}^{3}$ are synchronized as above, we expect the same $1 / 6$ supersymmetry is preserved by the Wilson loop. One can see the mechanism in the following way. Originally the supersymmetry parameters (and also the supercharges) constitute $(6,2)$ representation of $S U(4)_{R} \times S O(3)_{\text {Lorentz }}$. If we restrict to $S U(2)_{R} \subset S U(4)_{R}$, then the preserved supercharges are simply the singlets of the diagonal $S U(2) \subset S U(2)_{R} \times S O(3)_{\text {Lorentz }}$.

The choice of parameters $M(\tau), \hat{M}(\tau), \eta(\tau), \bar{\eta}(\tau)$ for arbitrarily shaped supersymmetric Wilson loop with $x(\tau)$ is reported in [14]. Instead of verifying the formulas of [14], in this paper we will establish evidences for supersymmetry by showing that the vacuum expectation value of such 1/6-BPS Wilson loops are trivial. In the forthcoming sections we construct explicitly a general class of such Wilson loops, and compute their expectation values perturbatively to check it is protected. 


\section{General 1/6-BPS Wilson loops}

As stated above, the idea is to relate $S U(2) \in S U(4)$ global R-symmetry of ABJM theory with the Lorentz group, and consider singlet of diagonal $S U(2)$. In particular, we allow the Wilson loop to take a generic contour in Euclidean spacetime $\mathbb{R}^{3}$. So $x^{\mu}(\tau)$ is an arbitrary 3 -vector at each $\tau$. We choose to denote it via $\tau$-dependent $S O(3)$ rotation from the BPS Wilson line of [11, in terms of Euler angles $\theta(\tau), \phi(\tau)$.

$$
v(\tau) \equiv \frac{\dot{x}(\tau)}{|\dot{x}(\tau)|}=R_{31}(\theta) R_{12}(\phi)\left(\begin{array}{l}
0 \\
0 \\
1
\end{array}\right)=\left(\begin{array}{c}
\sin \theta \cos \phi \\
\sin \theta \sin \phi \\
\cos \theta
\end{array}\right)
$$

Also we are to employ the same rotation for $z_{I}, \eta^{\alpha}$. In particular,

$$
\bar{\eta}(\tau)=e^{-i \phi \sigma_{3} / 2} e^{-i \theta \sigma_{2} / 2} \bar{\eta}(0)
$$

Here $\eta(0)=(1,0)^{T}$, and

$$
\eta^{\alpha}(\tau)=\sqrt{2}\left(\begin{array}{c}
e^{+i \phi / 2} \cos \frac{\theta}{2} \\
e^{-i \phi / 2} \sin \frac{\theta}{2}
\end{array}\right), \quad \bar{\eta}_{\alpha}(\tau)=\sqrt{2} i\left(\begin{array}{l}
e^{-i \phi / 2} \cos \frac{\theta}{2} \\
e^{+i \phi / 2} \sin \frac{\theta}{2}
\end{array}\right) .
$$

We rotate $z_{I}(I=1,2)$ in the same way as $\bar{\eta}_{\alpha}(\tau)$, i.e.

$$
z_{I}=\bar{z}^{I *}=\left(\begin{array}{llll}
e^{-i \phi / 2} \cos \frac{\theta}{2} & e^{+i \phi / 2} \sin \frac{\theta}{2} & 0 & 0
\end{array}\right)^{T}
$$

$M, \hat{M}$ are still defined according to $(6)$, and $\hat{M}=M^{T}=M^{*}$.

Let us here list a few useful properties of the parameters $M, \eta$ chosen as above:

$$
\begin{aligned}
\eta^{\alpha}(\tau) \bar{\eta}_{\alpha}(\tau) & =2 i \\
\left(\eta\left(v_{2}\right) \gamma^{\mu} \bar{\eta}\left(v_{1}\right)\right)\left(\eta\left(v_{1}\right) \bar{\eta}\left(v_{2}\right)\right) & =-2\left(v_{1}^{\mu}+v_{2}^{\mu}+i \epsilon_{\nu \rho}^{\mu} v_{1}^{\nu} v_{2}^{\rho}\right), \\
\operatorname{tr} M\left(\tau_{1}\right) M\left(\tau_{2}\right)=\operatorname{tr} \hat{M}\left(\tau_{1}\right) \hat{M}\left(\tau_{2}\right) & =2\left(1+v_{1} \cdot v_{2}\right) .
\end{aligned}
$$

\section{PERTURBATIVE CALCULATIONS}

Now let us move to the perturbative computations. For ABJM model the supersymmetric Wilson loops of a different class with $\operatorname{tr} M=0$ were studied in [9-11]. We choose to employ the conventions and results of [13. And we denote the $(n+1)$-th term in $(3)$ by $W^{(n)}$. 
The first term simply gives the dimensionalities of gauge groups, e.g. the top-left part is

$$
W_{\text {top-left }}^{(0)}=\operatorname{Tr}\langle 1\rangle=N
$$

So when we consider the entire supermatrix,

$$
W^{(0)}=N-M
$$

Because of supersymmetry this vacuum energy computation is exact to all orders.

Next, we consider the following

$$
\begin{aligned}
W_{t . l .}^{(1)} & =\operatorname{Tr} \int d \tau\left\langle i A_{\mu} \dot{x}^{\mu}+\frac{2 \pi}{k}|\dot{x}| M_{J}^{I} C_{I} \bar{C}^{J}\right\rangle \\
& =\frac{2 \pi}{k} \operatorname{Tr} \int d \tau|\dot{x}| M_{J}^{I}(\tau)\left\langle C_{I}(x(\tau)) \bar{C}^{J}(x(\tau))\right\rangle \\
& =\frac{2 \pi N M}{k}\left(\int d \tau|\dot{x}| \operatorname{tr} M\right) D(0) .
\end{aligned}
$$

Here we have denoted the scalar propagator by $D(x)$. More specifically,

$$
\left\langle C_{I}(x)_{i}^{\hat{j}} \bar{C}^{J}(y)_{\hat{k}}^{l}\right\rangle=\delta_{I}^{J} \delta_{i}^{l} \delta_{\hat{k}}^{\hat{j}} D(x-y), \quad D(x)=\frac{1}{4 \pi|x|} .
$$

We also made use of the fact that the one-point function of gauge field is zero. And we may also use the fact that $\operatorname{tr} M(\tau)=2$ for the class of supersymmetric Wilson loops we are interested in. So, up to this order, we have

$$
W^{(1)}=\frac{8 \pi N M}{k} L D(0) .
$$

where $L$ is the length of Wilson loop.

We then consider the next-order correction to this term. Obviously we should consider here the correction to the scalar field propator, but it is known to be zero for the ABJM mode due to supersymmetry.

Now let us move to the next order in the expansion of (3). We have

$$
W_{t . l .}^{(2)}=\int_{0}^{1} d \tau_{1} \int_{0}^{\tau_{1}} d \tau_{2}\left(-\left\langle\mathcal{A}\left(\tau_{1}\right) \mathcal{A}\left(\tau_{2}\right)\right\rangle+\frac{2 \pi}{k}\left|\dot{x}_{1}\right|\left|\dot{x}_{2}\right|\left\langle(\eta \bar{\psi})_{1}(\psi \bar{\eta})_{2}\right\rangle\right) .
$$

Here we denote as shorthand notation

$$
x_{1}=x\left(\tau_{1}\right), \quad x_{2}=x\left(\tau_{2}\right) \quad \text { etc. }
$$


We have several terms upon expansion, and for convenience we write them as follows and consider one by one.

$$
W_{t . l .}^{(2)}=\sum_{n=1}^{4} W_{n}^{(2)}
$$

where

$$
\begin{aligned}
W_{1}^{(2)}= & -\operatorname{Tr} \int_{0}^{1} d \tau_{1} \int_{0}^{\tau_{1}} d \tau_{2}\left\langle A_{\mu}\left(x_{1}\right) A_{\nu}\left(x_{2}\right)\right\rangle \dot{x}_{1}^{\mu} \dot{x}_{2}^{\nu} \\
W_{2}^{(2)}= & \frac{2 \pi i}{k} \operatorname{Tr} \int_{0}^{1} d \tau_{1} \int_{0}^{\tau_{1}} d \tau_{2}\left\langle A_{\mu}\left(x_{1}\right) C_{I}\left(x_{2}\right) \bar{C}^{J}\left(x_{2}\right)\right\rangle \dot{x}_{1}^{\mu}\left|\dot{x}_{2}\right| M_{J}^{I}\left(\tau_{2}\right)+(1 \leftrightarrow 2) \\
W_{3}^{(2)}= & \frac{4 \pi^{2}}{k^{2}} \operatorname{Tr} \int_{0}^{1} d \tau_{1} \int_{0}^{\tau_{1}} d \tau_{2} M_{J}^{I}\left(\tau_{1}\right) M_{L}^{K}\left(\tau_{2}\right)\left|\dot{x}_{1}\right|\left|\dot{x}_{2}\right| \\
& \times\left\langle C_{I}\left(x_{1}\right) \bar{C}^{J}\left(x_{1}\right) C_{K}\left(x_{2}\right) \bar{C}^{L}\left(x_{2}\right)\right\rangle \\
W_{4}^{(2)}= & \frac{2 \pi}{k} \operatorname{Tr} \int_{0}^{1} d \tau_{1} \int_{0}^{\tau_{1}} d \tau_{2}\left|\dot{x}_{1}\right|\left|\dot{x}_{2}\right| \eta_{I}^{\alpha}\left(\tau_{1}\right) \bar{\eta}\left(\tau_{2}\right)_{\beta}^{J}\left\langle\bar{\psi}_{\alpha}^{I}\left(x_{1}\right) \psi_{J}^{\beta}\left(x_{2}\right)\right\rangle .
\end{aligned}
$$

Now we need the propagator of the gauge fields. At tree level, it is given as follows

$$
\left\langle\left(A_{\mu}\right)_{i}^{j}(x)\left(A_{\nu}\right)_{k}^{l}(y)\right\rangle=\delta_{k}^{j} \delta_{i}^{l} \frac{i}{2 k} \epsilon_{\mu \nu \rho} \partial_{x}^{\rho} \frac{1}{|x-y|}
$$

Then we have

$$
W_{1 \text { tree }}^{(2)}=\frac{i N^{2}}{2 k} \int_{0}^{1} d \tau_{1} \int_{0}^{\tau_{1}} d \tau_{2} \frac{\epsilon_{\mu \nu \rho} \dot{x}_{1}^{\mu} \dot{x}_{2}^{\nu}\left(x_{1}-x_{2}\right)^{\rho}}{\left|x_{1}-x_{2}\right|^{3}} .
$$

This expression is zero for a planar contour, but in general it is not zero. This is related to the self-linking number of the contour (see e.g. [27] for further discussion), and an explicit computation requires regularization known as the framing procedure. This turns out to be cancelled by $W_{4}^{(2)}$, as we will explain later.

We are doing the computation up to $\mathcal{O}\left(k^{-2}\right)$ here, so we need to include the one-loop correction to 29$)$. This extra contribution is

$$
W_{\text {1oneloop }}^{(2)}=-\frac{N^{2} M}{k^{2}} \int_{0}^{1} d \tau_{1} \int_{0}^{\tau_{1}} d \tau_{2} \frac{\dot{x}_{1} \cdot \dot{x}_{2}}{\left|x_{1}-x_{2}\right|^{2}},
$$

up to a singular gauge transformation. For the one-loop vector field propagator in ChernSimons-matter theories, see e.g. [28].

We now turn to the next term. For this we need to know the cubic interaction vertex for two scalars and one vector. This comes from the gauge covariantized kinetic term of scalar fields. It is straightforward to read off the Feynman rule for vertex interaction, and 
the result is

$$
\begin{aligned}
\left\langle A_{\mu}(x) C_{I}(y) \bar{C}^{J}(z)\right\rangle= & \frac{i N^{2} M}{4 \pi^{2}} \delta_{I}^{J} \int d^{3} w\left(\epsilon_{\nu \mu \rho} \frac{i}{2 k} \partial_{w}^{\rho} \frac{1}{|w-x|} \frac{1}{|w-z|} \partial_{w}^{\nu} \frac{1}{|w-y|}\right. \\
& \left.-\epsilon_{\nu \mu \rho} \frac{i}{2 k} \partial_{w}^{\rho} \frac{1}{|w-x|} \frac{1}{|w-y|} \partial_{w}^{\nu} \frac{1}{|w-z|}\right) .
\end{aligned}
$$

Since in the Wilson loop computation we are to take $y=z$, it is obvious that $W_{2}^{(2)}=0$ [32].

We move on to the next term. Of course we consider 1PI diagrams only, and by contracting the flavor indices we have

$$
W_{3}^{(2)}=\frac{N^{2} M}{4 k^{2}} \int_{0}^{1} d \tau_{1} \int_{0}^{\tau_{1}} d \tau_{2} \frac{\left|\dot{x}_{1}\right|\left|\dot{x}_{2}\right| \operatorname{tr}\left(M\left(\tau_{1}\right) M\left(\tau_{2}\right)\right)}{\left|x_{1}-x_{2}\right|^{2}} .
$$

We need to know $\operatorname{tr} M_{1} M_{2}$ for supersymmetric Wilson loops, and as presented earlier (16), we may substitute

$$
\operatorname{tr} M_{1} M_{2}=2\left(1+\frac{\dot{x}_{1} \cdot \dot{x}_{2}}{\left|\dot{x}_{1}\right|\left|\dot{x}_{2}\right|}\right) .
$$

We turn to the last term at second order, for which we need the fermion propagator,

$$
\left\langle\psi_{I}(x) \bar{\psi}^{J}(y)\right\rangle=\frac{i}{4 \pi} \delta_{I}^{J} \gamma^{\mu} \partial_{x^{\mu}} \frac{1}{|x-y|} .
$$

So

$$
\begin{aligned}
W_{4}^{(2)} & =\frac{N M}{4 k} \int_{0}^{1} d \tau_{1} \int_{0}^{\tau_{1}} d \tau_{2}\left|\dot{x}_{1}\right|\left|\dot{x}_{2}\right|\left(\eta_{I}\left(\tau_{1}\right) \gamma^{\mu} \bar{\eta}^{I}\left(\tau_{2}\right)\right) \partial_{x_{1}^{\mu}} \frac{1}{\left|x_{1}-x_{2}\right|} \\
& =\frac{N M}{2 k} \int_{0}^{1} d \tau_{1} \int_{0}^{\tau_{1}} d \tau_{2}\left|\dot{x}_{1}\right|\left|\dot{x}_{2}\right|\left(\frac{\dot{x}_{1}}{\left|\dot{x}_{1}\right|}+\frac{\dot{x}_{2}}{\left|\dot{x}_{2}\right|}-i \frac{\dot{x}_{1} \times \dot{x}_{2}}{\left|\dot{x}_{1}\right|\left|\dot{x}_{2}\right|}\right) \cdot \frac{\left(x_{1}-x_{2}\right)}{\left|x_{1}-x_{2}\right|^{3}}
\end{aligned}
$$

The first two terms can be integrated once. Then one can easily check that these linearly divergent terms cancel the divergence of $W^{(1)}$ exactly. And the third term with $\dot{x}_{1} \times \dot{x}_{2}$ exactly cancels the self-linking number term $W_{1}^{(2)}$.

Collecting the results so far, we have

$$
W_{t . l .}^{(1)}+W_{t . l .}^{(2)}=-\frac{N M}{k} \int_{0}^{1} d \tau \frac{|\dot{x}(\tau)|}{|x(\tau)-x(0)|}+\frac{N^{2} M}{2 k^{2}} \int_{0}^{1} d \tau_{1} \int_{0}^{\tau_{1}} d \tau_{2} \frac{\left|\dot{x}_{1}\right|\left|\dot{x}_{2}\right|-\dot{x}_{1} \cdot \dot{x}_{2}}{\left|x_{1}-x_{2}\right|^{2}} .
$$

This is the result for the $N \times N$ block, and for the final answer we should include the $M \times M$ block too. Upon taking the supertrace, the first term cancels and the second term is nonzero in general, but also vanishes for the ABJM model, i.e. if $N=M$. Recapitulating, we have established that up to $\mathcal{O}\left(1 / k^{2}\right)$ the expectation value of our Wilson loops vanishes for the ABJM model. 


\section{WORLDSHEET SOLUTION AND PSEUDO-HOLOMORPHICITY}

In this section we will present the string theory side description of the generic contour Wilson loop we considered in the previous sections. Let us start with the IIA supergravity solution $A d S_{4} \times \mathbb{C P}^{3}$, with metric

$$
d s_{10}^{2}=\frac{R^{3}}{4 k}\left(d s_{A d S_{4}}^{2}+4 d s_{\mathbb{C P}^{3}}^{2}\right) .
$$

We use the usual Poincare coordinates for $A d S_{4}$,

$$
d s_{A d S_{4}}^{2}=r^{2}\left(-d t^{2}+d x^{2}+d y^{2}\right)+\frac{d r^{2}}{r^{2}}
$$

And for $\mathbb{C P}^{3}$ we take the following parametrization,

$$
\begin{aligned}
d s_{\mathbb{C P}^{3}}^{2}= & \frac{1}{4}\left[d \alpha^{2}+\cos ^{2} \frac{\alpha}{2}\left(d \theta_{1}^{2}+\sin ^{2} \theta_{1} d \varphi_{1}^{2}\right)+\sin ^{2} \frac{\alpha}{2}\left(d \theta_{2}^{2}+\sin ^{2} \theta_{2} d \varphi_{2}^{2}\right)\right. \\
& \left.+\sin ^{2} \frac{\alpha}{2} \cos ^{2} \frac{\alpha}{2}\left(d \chi+\cos \theta_{1} d \varphi_{1}-\cos \theta_{2} d \varphi_{2}\right)^{2}\right] .
\end{aligned}
$$

Here $R$ sets the radius of curvature, and $k$ is related to the order of the orbifolding action [6].

In order to check the supersymmetry of probe strings, we need the Killing spinor and its supersymmetry projection rule. We will study fundamental string configuration which is confined to the subspace $A d S_{4} \times S^{2}$, where the two-sphere is parametrized by $\theta_{1}, \varphi_{1}$. For

simplicity we set the rest of parameters to zero. After renaming $\theta_{1}=\theta, \varphi_{1}=\phi$, the Killing spinor in $A d S_{4} \times S^{2}$ is reduced to (we follow the convention in e.g. [29])

$$
\begin{aligned}
\epsilon & =e^{\frac{\theta}{4}\left(\hat{\Gamma} \Gamma_{5}-\Gamma_{6} \Gamma_{11}\right)} e^{\frac{\phi}{4}\left(\Gamma_{56}-\Gamma_{11} \hat{\Gamma}\right)} \\
& \times\left\{1+\frac{r}{2}\left(t \Gamma^{03}\left(1-\Gamma^{012}\right)-x \Gamma^{13}\left(1-\Gamma^{012}\right)-y \Gamma^{23}\left(1-\Gamma^{012}\right)\right)\right\} r^{\frac{\Gamma^{012}}{2}} \epsilon_{0} .
\end{aligned}
$$

Here and below we use frame indices for gamma matrices. The ordering we use is $t, x, y, r, \alpha, \theta_{1}, \varphi_{1}, \theta_{2}, \varphi_{2}, \chi$. Then the prjection rule for 24 out of 32 spinors is given as

$$
\left(\Gamma^{0123}+\left(\Gamma^{49}+\Gamma^{56}+\Gamma^{78}\right) \Gamma_{11}\right) \epsilon=0 .
$$

This condition can be easily obtained from the dilatino variation of IIA supergravity. Since (42) should be satisfied at every point of the spacetime, one should demand it on $\epsilon_{0}$ as well.

The $\kappa$-symmetry prescription requires that $\Gamma_{\kappa}$ projection defined through the induced worldvolume form should be compatible with the Killing spinor of the background (41). As 
a warm-up one may start with string stretched along $r$-direction. Obviously $\Gamma_{\kappa}=\Gamma^{03}$. If we set for simplicity $\theta=\phi=x=y=0$, the Killing spinor is simplified to

$$
\epsilon=\left[1+\frac{r t}{2} \Gamma^{03}\left(1-\Gamma^{012}\right)\right] r^{\frac{\Gamma^{012}}{2}} \epsilon_{0}
$$

Then one can check $\Gamma^{03} \Gamma_{11} \epsilon= \pm \epsilon$ is satisfied if we impose the same projection $\Gamma^{03} \Gamma_{11} \epsilon_{0}=$ $\pm \epsilon_{0}$, so this configuration is $1 / 2-\mathrm{BPS}$.

Now we consider a Zarembo-like solution with circular contour in both a spatial subspace of $A d S_{4}$ and $S^{2}$. We set $t=0$ and using $z, \sigma$ as worldsheet coordinates the solution is

$$
\begin{aligned}
& x=-\sqrt{\rho^{2}-z^{2}} \sin \sigma, \quad y=\sqrt{\rho^{2}-z^{2}} \cos \sigma, \quad r=1 / z, \\
& \theta=\tan ^{-1}\left(r \sqrt{\rho^{2}-z^{2}}\right), \quad \phi=\sigma .
\end{aligned}
$$

On the boundary the string worldsheet is a circle with radius $\rho$. This configuration satisfies the string equation of motion and in particular it is pseudo-holomorphic in the sense of [15]. In order to check that, we first note that the induced metric and Kähler form on the worldsheet are

$$
d s_{w s}^{2}=\frac{z^{2}+\rho^{2}}{z^{2}\left(\rho^{2}-z^{2}\right)} d z^{2}+\frac{\rho^{4}-z^{4}}{z^{2} \rho^{2}} d \sigma^{2}, \quad j_{w s}=\frac{\rho^{2}+z^{2}}{z^{2} \rho} d z \wedge d \sigma .
$$

To endow a complex structure to $A d S_{4} \times S^{2}$ it is advantageous to write the metric in the following way.

$$
\begin{aligned}
d s^{2} & =\frac{R^{3}}{4 k}\left[r^{2}\left(-d t^{2}+d x^{2}+d y^{2}\right)+\frac{d r^{2}}{r^{2}}+d \theta^{2}+\sin ^{2} \theta d \phi^{2}\right] \\
& =\frac{R^{3}}{4 k}\left[\left(X^{2}+Y^{2}+Z^{2}\right)\left(-d t^{2}+d x^{2}+d y^{2}\right)+\frac{d X^{2}+d Y^{2}+d Z^{2}}{X^{2}+Y^{2}+Z^{2}}\right] .
\end{aligned}
$$

We introduced $\mathbb{R}^{3}$ coordinates $X, Y, Z$ from $r, \theta, \phi$ in the obvious way. The complex structure in the target space of our choice is so that $J=d x \wedge d X+d y \wedge d Y+d t \wedge d Z$. It is then straightforward to check the pseudo-holomorphicity condition $j_{w s} \cdot P \cdot J=P$ with $P_{\alpha}^{M}=\partial_{\alpha} X^{M}$.

Now we can compute $\Gamma_{\kappa}$, using the induced volume form on the worldsheet.

$$
\begin{aligned}
\Gamma_{\kappa}= & i \frac{\rho z^{2}}{\rho^{2}+z^{2}} \Gamma_{11}\left[-\frac{1}{z} \Gamma_{12}+\frac{1}{\rho}\left(\sin \sigma \Gamma_{1}-\cos \sigma \Gamma_{2}\right) \Gamma_{6}\right. \\
& \left.+\left(\frac{\sqrt{\rho^{2}-z^{2}}}{z} \Gamma_{3}+\Gamma_{5}\right)\left(\frac{1}{z}\left(\cos \sigma \Gamma_{1}+\sin \sigma \Gamma_{2}\right)-\frac{1}{\rho} \Gamma_{6}\right)\right] .
\end{aligned}
$$


The problem is then to check if there exists a set of projection conditions on constant spinor $\epsilon_{0}$, upon which the supersymmetry condition $\Gamma_{\kappa} \epsilon=\epsilon$ is satisfied at every point $(z, \sigma)$. Since the form of $\Gamma_{\kappa}$ is quite involved, this is a nontrivial task.

Our strategy is first to make an educated guess for (part of) the projection rule, which simplifies the form of Killing spinor 411. Then we derive the rest of projection rule at a special point on the worldsheet where $\Gamma_{\kappa}$ is simplified. In the end we will see that our circular Wilson loop configuration is $1 / 6-\mathrm{BPS}$.

The hint for the projection rule is that we have chosen 5,6 directions as the $S^{2}$ part of the active metric. This combined with the SUSY rule (42) imply that on Killing spinor we should impose

$$
\left(\Gamma^{49}+\Gamma^{78}\right) \epsilon_{0}=\left(\Gamma^{56}+\hat{\Gamma} \Gamma_{11}\right) \epsilon_{0}=0 .
$$

These are compatible with the 3/4-BPS condition 42, and one can easily check that the three of them together constitute 1/2-BPS condition $\epsilon_{0}$. With these conditions, we may rewrite (41) as

$$
\begin{aligned}
\epsilon & =\left\{1+\frac{r}{2}\left(t \Gamma^{03}\left(1-\Gamma^{012}\right)-x \Gamma^{13}\left(1-\Gamma^{012}\right)-y \Gamma^{23}\left(1-\Gamma^{012}\right)\right)\right\} r^{\frac{\Gamma^{012}}{2}} \\
& \times\left(\cos \frac{\phi}{2} e^{\frac{\theta}{2} \hat{\Gamma} \Gamma_{5}}+\Gamma_{56} \sin \frac{\phi}{2} e^{-\frac{\theta}{2} \hat{\Gamma} \Gamma_{5}}\right) \epsilon_{0} .
\end{aligned}
$$

To infer the rest of the projection rule, let us consider a specific point on worldvolume first, e.g. $z=\rho, \sigma=0$. Then we have

$$
i \Gamma_{\kappa} \Gamma_{11}=\Gamma_{12}+\Gamma_{26}+\Gamma_{15}+\Gamma_{56} .
$$

For the Killing spinor, let us further set $t=x=y=0$ for simplicity. Then

$$
\epsilon=\rho^{-\frac{\Gamma^{012}}{2}} \epsilon_{0} .
$$

And it is easy to calculate that $\Gamma_{\kappa} \epsilon=\epsilon$ leads to

$$
\begin{aligned}
\epsilon_{0} & =\rho^{+\frac{\Gamma^{012}}{2}} \frac{i \Gamma_{11}}{2}\left(\Gamma_{12}+\Gamma_{26}+\Gamma_{15}+\Gamma_{56}\right) \rho^{-\frac{\Gamma^{012}}{2}} \epsilon_{0} \\
& =\frac{i \Gamma_{11}}{2}\left(\left(\Gamma_{12}+\Gamma_{56}\right) \rho^{-\Gamma^{012}}+\left(\Gamma_{26}+\Gamma_{15}\right)\right) \epsilon_{0} .
\end{aligned}
$$

In order to satisfy this for arbitrary $\rho$ it is then required

$$
\Gamma_{1256} \epsilon_{0}=\epsilon_{0} .
$$


And when we combine this condition with the background projection rule (42), it is clear that we should also impose

$$
\epsilon_{0}=i \Gamma_{11} \Gamma_{15} \epsilon_{0}
$$

The projection rules we have identified so far leave 4 linearly independent spinors, thus make our solution 1/6-BPS of the background. We have also checked that for such Killing spinors the $\Gamma_{\kappa}$ projection is satisfied at every point of the worldvolume, by explicitly evaluating $\left(\Gamma_{\kappa}-1\right) \epsilon$ using a concrete basis for gamma matrices. One possible loophole of our argument so far is that $\Gamma_{4789} \epsilon_{0}=\epsilon_{0}$ is rather ad hoc. However we have also verified that it is impossible to satisfy the $\Gamma_{\kappa}$ projection everywhere on the worldsheet if we consider the other half of the spinors $\Gamma_{4789} \epsilon_{0}=-\epsilon_{0}$.

\section{DISCUSSIONS}

We have studied supersymmetric Wilson loops in ABJM model, in particular the ones with generic contours in this paper. Their existence and the conserved supersymmetries are nontrivial on both sides of the AdS/CFT correspondence. We have given explicit construction of Wilson loops both in ABJM model and the dual string theory, and checked the supersymmetry and their expectation values. We believe our analysis in this paper provides another nontrivial evidence for the ABJM conjecture.

Being $(N+M) \times(N+M)$, the supermatrix in (2) can be considered as in fundamental representation of $S U(N \mid M)$. For the case of $\mathcal{N}=4$ super Yang-Mills and IIB string theory in $A d S_{5} \times S^{5}$, it is known that Wilson loops in generic tensor representations are dual to D3 or D5 branes [30] 31]. It is natural to expect that string-like D-branes in $A d S_{4} \times \mathbb{C P}^{3}$ described e.g. in [16] are dual to Wilson loops in different representations of supergroup $S U(N \mid M)$. It must be interesting to construct such operators in ABJM model and to calculate them in perturbation theory. We plan to address these issues in forthcoming publications.

\section{Acknowledgments}

We thank Jun-Bao Wu for useful comments. NK gratefully acknowledges support from the Institute for Advanced Study, where part of this work was carried out. This work is supported by the sabbatical leave petition program (2012) of Kyung Hee University, the 
National Research Foundation of Korea (NRF) funded by the Ministry of Education, Science and Technology (MEST) of Korea with grant No. 670522, 2010-0023121, 2012046278 and also through the Center for Quantum Spacetime (CQUeST) of Sogang University with grant No. 2005-0049409.

[1] J. M. Maldacena, The Large N limit of superconformal field theories and supergravity, Adv.Theor.Math.Phys. 2 (1998) 231-252, hep-th/9711200.

[2] J. M. Maldacena, Wilson loops in large N field theories, Phys.Rev.Lett. 80 (1998) 4859-4862, hep-th/9803002.

[3] S.-J. Rey and J.-T. Yee, Macroscopic strings as heavy quarks in large $N$ gauge theory and anti-de Sitter supergravity, Eur.Phys.J. C22 (2001) 379-394, hep-th/9803001.

[4] J. Erickson, G. Semenoff, and K. Zarembo, Wilson loops in N=4 supersymmetric Yang-Mills theory, Nucl.Phys. B582 (2000) 155-175, hep-th/0003055.

[5] V. Pestun, Localization of gauge theory on a four-sphere and supersymmetric Wilson loops, Commun.Math.Phys. 313 (2012) 71-129, arXiv:0712.2824.

[6] O. Aharony, O. Bergman, D. L. Jafferis, and J. Maldacena, N=6 superconformal Chern-Simons-matter theories, M2-branes and their gravity duals, JHEP 0810 (2008) 091, arXiv:0806.1218.

[7] O. Aharony, O. Bergman, and D. L. Jafferis, Fractional M2-branes, JHEP 0811 (2008) 043, arXiv:0807.4924.

[8] N. Drukker, J. Plefka, and D. Young, Wilson loops in 3-dimensional N=6 supersymmetric Chern-Simons Theory and their string theory duals, JHEP 0811 (2008) 019, arXiv:0809.2787.

[9] B. Chen and J.-B. Wu, Supersymmetric Wilson Loops in N=6 Super Chern-Simons-matter theory, Nucl.Phys. B825 (2010) 38-51, arXiv:0809.2863.

[10] S.-J. Rey, T. Suyama, and S. Yamaguchi, Wilson Loops in Superconformal Chern-Simons Theory and Fundamental Strings in Anti-de Sitter Supergravity Dual, JHEP 0903 (2009) 127, arXiv:0809.3786.

[11] N. Drukker and D. Trancanelli, A Supermatrix model for N=6 super Chern-Simons-matter theory, JHEP 1002 (2010) 058, arXiv:0912.3006. 
[12] K. Zarembo, Supersymmetric Wilson loops, Nucl.Phys. B643 (2002) 157-171, hep-th/0205160.

[13] L. Griguolo, D. Marmiroli, G. Martelloni, and D. Seminara, The generalized cusp in ABJ(M) $N=6$ Super Chern-Simons theories, arXiv:1208.5766.

[14] V. Cardinali, L. Griguolo, G. Martelloni, and D. Seminara, New supersymmetric Wilson loops in ABJ(M) theories, Phys.Lett. B718 (2012) 615-619, arXiv:1209.4032.

[15] A. Dymarsky, S. S. Gubser, Z. Guralnik, and J. M. Maldacena, Calibrated surfaces and supersymmetric Wilson loops, JHEP 0609 (2006) 057, hep-th/0604058.

[16] J. Kluson and K. L. Panigrahi, Defects and Wilson Loops in 3d QFT from D-branes in $A d S(4) x C P^{* * 3}, \operatorname{arXiv:0809.3355.~}$

[17] J. Kluson and K. L. Panigrahi, Wilson Loops from D-branes in AdS(4) X CP**3 with B(NS) Holonomy, Eur.Phys.J. C67 (2010) 565-573, arXiv:0906.2148.

[18] J. M. Henn, J. Plefka, and K. Wiegandt, Light-like polygonal Wilson loops in 3d Chern-Simons and ABJM theory, JHEP 1008 (2010) 032, arXiv:1004.0226.

[19] K.-M. Lee and S. Lee, 1/2-BPS Wilson Loops and Vortices in ABJM Model, JHEP 1009 (2010) 004, arXiv:1006.5589.

[20] M. S. Bianchi, M. Leoni, A. Mauri, S. Penati, C. Ratti, et. al., From Correlators to Wilson Loops in Chern-Simons Matter Theories, JHEP 1106 (2011) 118, arXiv:1103.3675.

[21] K. Wiegandt, Equivalence of Wilson Loops in $\mathcal{N}=6$ super Chern-Simons matter theory and $\mathcal{N}=4$ SYM Theory, Phys.Rev. D84 (2011) 126015, arXiv:1110.1373.

[22] H. Kim, N. Kim, and J. H. Lee, One-loop corrections to holographic Wilson loop in AdS4xCP3, J.Korean Phys.Soc. 61 (2012) 713-719, arXiv:1203.6343.

[23] A. Klemm, M. Marino, M. Schiereck, and M. Soroush, ABJM Wilson loops in the Fermi gas approach, arXiv:1207.0611.

[24] D. Marmiroli, Resumming planar diagrams for the $N=6$ ABJM cusped Wilson loop in light-cone gauge, arXiv:1211.4859.

[25] M. S. Bianchi, G. Giribet, M. Leoni, and S. Penati, The 1/2 BPS Wilson loop in ABJM theory at two loops, arXiv:1303.6939.

[26] M. S. Bianchi, G. Giribet, M. Leoni, and S. Penati, Light-like Wilson loops in ABJM and maximal transcendentality, arXiv:1304.6085

[27] E. Guadagnini, M. Martellini, and M. Mintchev, Wilson Lines in Chern-Simons Theory and 
Link Invariants, Nucl.Phys. B330 (1990) 575.

[28] D. Gaiotto and X. Yin, Notes on superconformal Chern-Simons-Matter theories, JHEP 0708 (2007) 056, arXiv:0704.3740.

[29] H. Kim and N. Kim, On the supersymmetry of spiky branes in $A d S(4) x C P^{* *}$, Mod.Phys.Lett. A25 (2010) 3267-3277.

[30] S. Yamaguchi, Wilson loops of anti-symmetric representation and D5-branes, JHEP 0605 (2006) 037, hep-th/0603208.

[31] J. Gomis and F. Passerini, Holographic Wilson Loops, JHEP 0608 (2006) 074, hep-th/0604007.

[32] This term was argued to be zero in previous papers, but for different reasons. Rey et. al. [10] noticed this term in the end should involve $\operatorname{tr} M$ which is zero for the 1/6-BPS Wilson loop they were considering. On the other hand, Drukker, Plefka and Young [8] pointed out this term vanishes for planar Wilson loop, irrespective of the value of $\operatorname{tr} M$. 